Revisión

\title{
Tomoterapia helicoidal: IMRT adaptada guiada por imagen
}

\author{
D. Ortiz de Urbina, J. M. Delgado
}

\section{Resumen}

El Tomo-Therapy HI-ART es un nuevo sistema de radioterapia que integra la planificación del tratamiento, la verificación de la posición del paciente, del target y de los órganos a riesgo (OAR) y la ejecución de la administración de la radiación. Mediante un colimador multiláminas binario es posible modular la intensidad de un haz de radiación helicoidal, rotatorio y continuo, generado en un Acelerador Lineal instalado en un gantry de morfología anular, obteniendo altos índices de conformabilidad en el target, distribuciones de dosis homogéneas y altos gradientes dosimétricos, optimizando el rango terapéutico.

Además, proporciona imágenes volumétricas de TAC (tomoimagen) de los volúmenes de interés (target, OAR) inmediatamente antes del tratamiento, permitiendo en tiempo real certificar la posición del paciente, precisar con exactitud la localización del target y de los OAR, y adaptar el tratamiento de radioterapia de acuerdo a las posibles modificaciones que ocurren durante el tiempo de tratamiento en la morfología, tamaño y movilidad del tumor.

En conclusión, Tomo-Therapy HI-ART integra en una única unidad funcional tratamiento e imagen utilizando un Acelerador Lineal instalado en un gantry anular helicoidal, siendo una modalidad de alta precisión de radioterapia de intensidad modulada (IMRT) guiada por imagen tomográfica.

\section{Palabras clave:}

Tomoterapia. IMRT. Radioterapia adaptada.

Oncología, 2005; 28 (8):367-377 


\section{Summary}

The Tomo-Therapy HI-ART System delivers intensity-modulated radiotherapy (IMRT) using a multileaf collimator (MLC). This binary MLC is made up of interlaced leaves, where each leaf can open and close quickly to permit or block the radiation throughout it, dividing the radiation beam into many smaller beams (beamlets).

The Tomo-Therapy System also includes a computed tomography (CT) which can verify the size, shape and location of the patient's anatomy, target and organs at risk just prior to the time of treatments.

This integrated system allows to optimize the planification, CT verification and fusion images, set-up modifications on line, and finally to deliver the dose, verify the dose delivered and made a dose reconstruction, a whole process called adaptive radiotherapy.

The Tomo-Therapy is the first system of image CT-guided IMRT.

Key words: Tomo-Therapy. IMRT. Adaptive Radiotherapy.

\section{Introducción}

En la última década, la introducción de nuevas tecnologías, han permitido incorporar sofisticadas técnicas de irradiación, que han tenido por objeto conseguir altos índices de conformación geométrica ajustadas a las diferentes morfologías de los volúmenes tumorales, y distribuciones de alto gradiente de dosis con el objeto de administrar una dosis eficaz en el tumor evitando y/o minimizando la radiación innecesaria en las estructuras anatómicas correspondientes a los tejidos sanos inmediatamente adyacentes. En este sentido, han sido desarrolladas para la práctica clínica la radiocirugía estereotáxica, la radioterapia estereotáxica fraccionada, la radioterapia conformada tridimensional (3D) y la radioterapia de intensidad modulada (IMRT).

A mediados de los años 90, se populariza la técnica de IMRT cuando NOMOS Corporation (Pennsylvania, USA) introduce el sistema PEACOCK, que incluye un colimador multiláminas terciario (MIMIC) y un sistema de planificación inverso, precipitando la administración de tratamientos con radiación caracterizados por la utilización de un número de arcos discreto, de diferente amplitud, entre los cuales la mesa de tratamiento se mueve longitudinalmente, definiendo la denominada Tomoterapia en serie.

El mayor handicap de la IMRT consiste en la incertidumbre geométrica relacionada con la posición relativa y con la morfología del tumor y de los OAR en cada sesión de tratamiento; cualquier error en este sen- tido provocaría un riesgo de infradosificación del volumen de tratamiento definido (GTV, CTV, PTV) y/o una sobredosificación de tejidos normales adyacentes.

Por dicho motivo, se han introducido técnicas de radioterapia guiadas por imagen (IGT), que tienen por objeto identificar las estructuras anatómicas mediante la adquisición de imágenes volumétricas durante el tratamiento, bien instalando en la misma sala de tratamiento dos unidades independientes, TAC (imagen) y Acelerador Lineal (tratamiento), que emplean una mesa de tratamiento común o bien el "cone-beam" que incorpora un sistema de imagen de kilovoltaje (TAC) colocado ortogonalmente respecto al Acelerador Lineal ${ }^{1-3}$.

En el año 1993, Mackie y cols ${ }^{4}$ introducen la unidad de radiación denominada Tomo-Therapy Inc. HI-ART (Madison, WI), o unidad de tomoterapia helicoidal, que integra por primera vez un sistema de radioterapia guiado por TAC. La tomoterapia helicoidal se define como una nueva técnica de administración de radiación de intensidad modulada (IMRT) mediante un haz rotatorio ("Fan beam") generado en un Acelerador Lineal instalado en un gantry anular similar al gantry de un TAC, que emite radiación de forma continua y simultáneamente mientras la mesa de tratamiento desplaza longitudinalmente al paciente a través del haz de radiación.

La tomoterapia helicoidal es una tomo-radiación administrada con un movimiento simultáneo del gantry y de la mesa de tratamiento asimilado conceptualmente a un TAC helicoidal. 


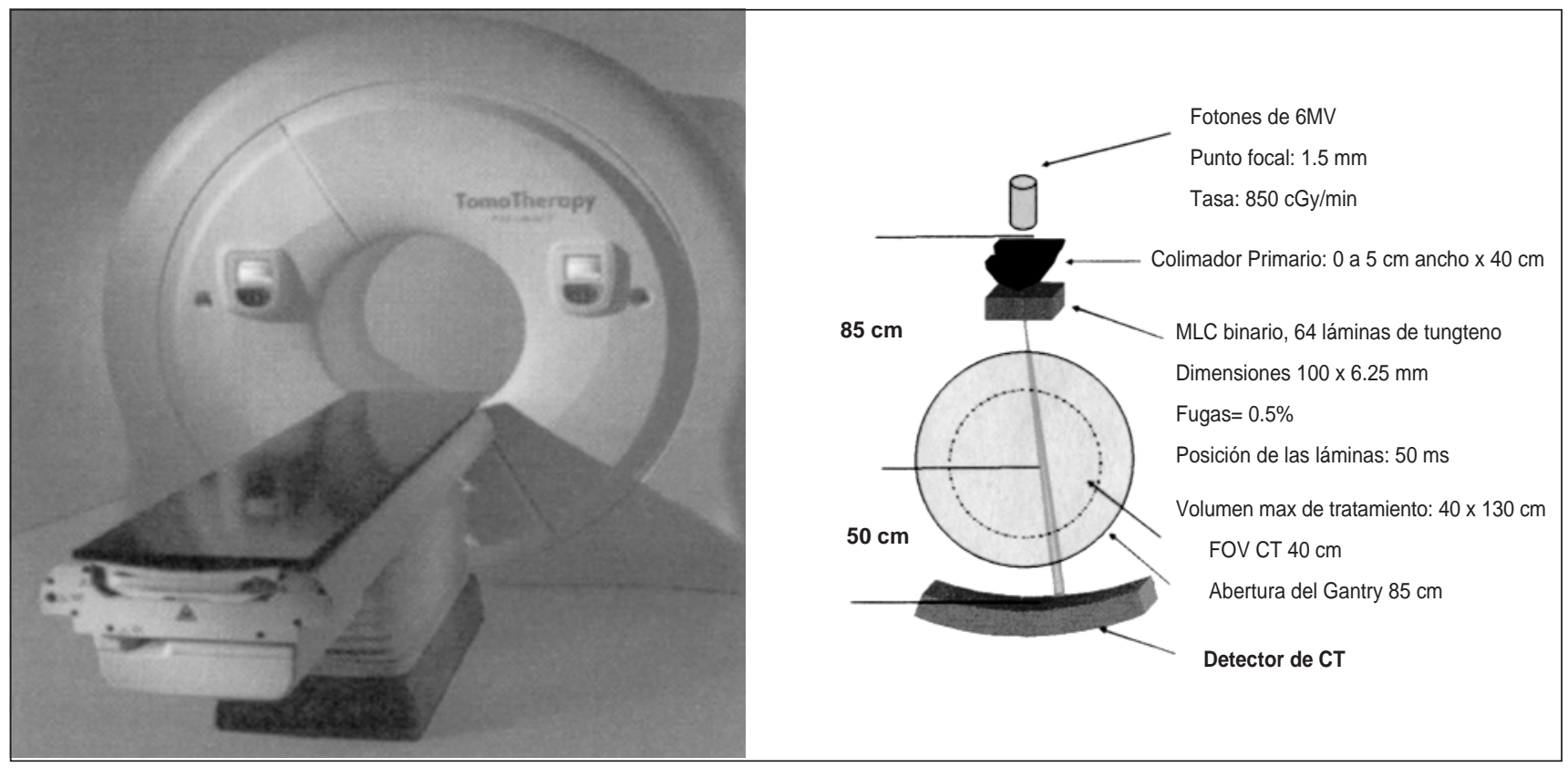

Figura 1. Tomografía Hi-Art desarrollado en la Universidad de Wisconsin.

La utilización del Acelerador Lineal como fuente de rayos $\mathrm{X}$ de bajo megavoltaje genera una tomoimagen que permite la localización exacta del "target" en el paciente inmediatamente antes de su tratamiento.

\section{Unidad de tomoterapia HI-ART: descripción (Figura 1)}

Se trata del único sistema de tomoterapia helicoidal de uso clínico que integra un Acelerador Lineal que opera con fotones de $6 \mathrm{MV}$ y un sistema de detección en la salida del haz de radiación que genera una imagen tipo TAC con fotones de $3.5 \mathrm{MV}^{5,6}$.

Un sistema de mandíbulas independiente integrado con el colimador primario, produce el "Fan beam" con una amplitud del haz entre 1 y $5 \mathrm{~cm}$. Además, un colimador multiláminas binario, compuesto por 64 láminas, interacciona a través del haz de radiación mediante un sistema de apertura/cierre definiendo pequeños haces ("beamlets") individuales, siendo el tiempo de apertura/cierre de las láminas de aproximadamente 50 milisegundos; el haz de radiación reproduce entre 2 a 5 rotaciones en cada punto o lo que es lo mismo entre 100-250 beamlets, de manera que varios miles de beamlets son utilizados en cada tratamiento.
La distancia de la fuente de radiación al eje de rotación es de $85 \mathrm{~cm}$, y hace factible tratar un volumen cilíndrico de $40 \mathrm{~cm}$ de diámetro por $160 \mathrm{~cm}$ de longitud en un único tiempo de radiación, con una tasa de dosis de $850 \mathrm{cGy} /$ minuto.

El método de cálculo de dosis es de convolución/ superposición y un sofisticado sistema informático compuesto por un total de 32 procesadoras-computadoras es responsable del cálculo de dosis, su optimización y el almacenamiento en memoria de los datos calculados.

La integración de un Acelerador Lineal en un gantry anular tipo TAC proporciona una serie de ventajas:

- Similar al TAC convencional, el gantry anular del sistema Tomo-Therapy HI-ART reproduce una precisión en el isocentro del orden de décimas de $\mathrm{mm}$, que se compara favorablemente con $1 \mathrm{~mm}$ con el gantry convencional de los Aceleradores Lineales.

- La fuente de rayos X utilizada para generar la imagen es el Acelerador Lineal, utilizando un haz menor de energía y fluencia; la utilización del mismo haz de tratamiento sin modificar su trayectoria asegura que la tomoimagen coincida exactamente con el volumen diana radiado en tiempo real. 
- La unidad de tomoterapia genera secciones de TAC cada 5 segundos y permite discriminar con nitidez las diferentes estructuras anatómicas (pulmón, grasa, músculo, hueso).

- Una dosis de apenas 0,5-1,5 cGy es suficiente para visualizar estructuras en la tomoimagen, resultando una dosis mucho más baja que la dosis recibida utilizando sistemas de imagen por TAC convencional.

\section{Tomoterapia: radioterapia adaptada}

La utilización de imágenes de TAC en la planificación del tratamiento de radioterapia, puede contribuir a asumir una falsa sensación de precisión, si no tenemos en cuenta que el diseño y la estrategia del tratamiento así como la elección definitiva del mismo se fundamentan en una realidad anatómica en el momento puntual de la realización del estudio. En general, los tratamientos con radiación contemplan una duración de varias semanas y son divididos en diferentes fracciones administrando una dosis diaria (fraccionamiento convencional) o varias dosis diarias (hiperfraccionamiento). Durante dichas semanas, la anatomía del paciente, la morfología y el tamaño del tumor y la movilidad de las estructuras anatómicas se modifican, de manera que la estimación de un correcto tratamiento en la planificación previa al inicio de la radiación puede ser insatisfactoria en el transcurso de la misma y conllevar importantes errores que condicionen la infradosificación del volumen tumoral (recidiva tumoral) o la sobredosificación de órganos de riesgo (toxicidad), aspecto especialmente a considerar cuando utilizamos técnicas de radiación altamente conformadas (IMRT, RT estereotáxica, RTC-3D).

La optimización de un tratamiento con radioterapia requiere, además de conseguir distribuciones espaciales geométricas complejas de dosis, que dicha dosis sea administrada con seguridad y precisión en el target, a la vez que evitada en los OAR, siendo esto posible solamente mediante la adquisición de una imagen del paciente y del target inmediatamente antes de la administración de cada fracción de tratamiento.

El prototipo de tomoterapia desarrollado en la Universidad de Wisconsin integra además de un Acelerador Lineal, un colimador multiláminas binario, un detector que recoge la salida del haz de ra- diación y un sistema de imagen tomográfica computarizada. El detector de CT localizado del lado opuesto al Acelerador Lineal, y en la misma trayectoria del haz de radiación, permite adquirir imágenes de TAC de megavoltaje (tomoimagen) además de verificar las señales recogidas durante el tratamiento que permiten confirmar que la radiación recibida por el paciente está de acuerdo con la radiación prevista?.

Es fácil entender que la base de los procesos de verificación radica en la capacidad de la Unidad de Tomoterapia para generar imagen de CT en tiempo real $^{8}$.

El procedimiento de radioterapia adaptada comienza con la adquisición de imágenes del paciente en 3D y posterior definición de las estructuras de interés mediante el contorneo del tumor y de las estructuras anatómicas críticas y/o radiosensibles. Posteriormente, se procede al desarrollo y optimización de la planificación del tratamiento, que en el caso de la tomoterapia utiliza un sistema de planificación inversa, y cuyo objetivo es conseguir una distribución de dosis en el contexto de un tratamiento basado en IMRT 9 .

Previo a la realización del tratamiento, el paciente es colocado reproduciendo las condiciones de inmovilización y posición previamente consideradas, y a través de una tomoimagen, se determina sí el paciente está colocado adecuadamente. Una técnica de fusión de imagen automática permite solapar las imágenes TAC de planificación con las imágenes actuales de megavoltaje de la tomoterapia y comprobar que tanto el paciente como las estructuras internas (target, OAR,...) están correctamente posicionadas, a la vez que identificar si han ocurrido modificaciones en el tamaño y/o la forma del tumor o de la anatomía del paciente ${ }^{10,11}$. En el caso de existir discrepancias, es posible reposicionar al paciente de forma automática modificando los parámetros de rotación o traslación hasta conseguir su ajuste de acuerdo a la posición planificada, comprobando al mismo tiempo que la posición del target y los OAR no han experimentado una desviación relativa al marco de referencia del paciente (Figura 2). La corrección de dicha discrepancia puede realizarse desplazando al paciente hasta la posición correspondiente a la de radiación o modificando y ajustando la planificación del tratamiento de acuerdo a la nueva posición del paciente. De esta manera es posible 


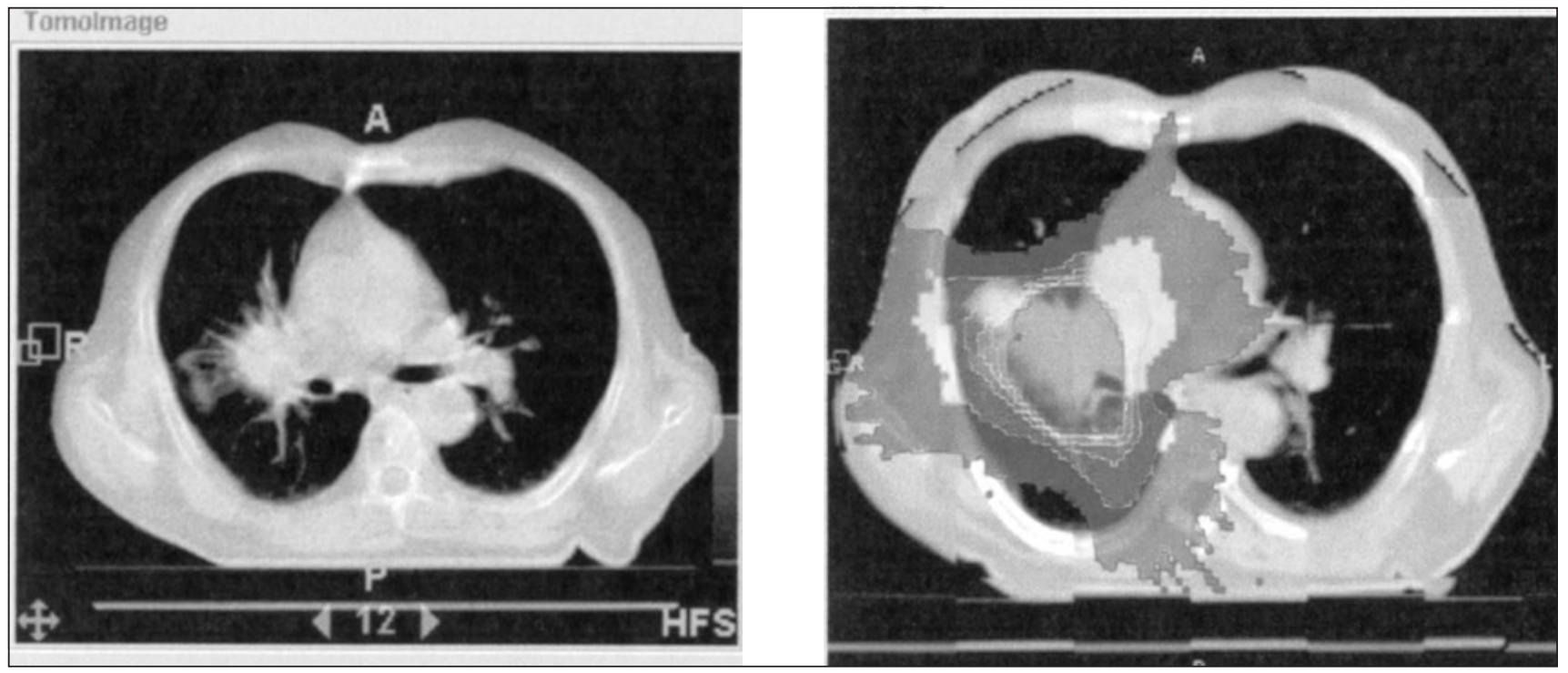

Figura 2. Estudio de verificación y fusión de imagen Kilovoltaje-TAC y Megalvoltaje-TAC en ca. no microcítico de pulmón. (Hospital San Raffaelle, Milan).

asegurar que el tumor está en condiciones de recibir la radiación prevista ${ }^{12}$.

La Unidad de Tomoterapia permite la verificación de la dosis mediante un detector de megavoltaje que recoge la cantidad de radiación transmitida a través del paciente durante el tratamiento; dichas señales pueden ser comparadas con las señales previstas de manera anticipada, siendo posible la interrupción del tratamiento si existen incertidumbres o discrepancias en las mismas.

Las señales detectadas en el proceso de verificación de la dosis administrada son de gran utilidad para conocer la cantidad de radiación incidente en el paciente ${ }^{13,14}, y$, combinada con la tomoimagen realizada durante el tratamiento proporciona una importante información que tiene que ver con la reconstrucción de la dosis ${ }^{15}$. En una situación ideal, si la anatomía del paciente no cambia, los parámetros de colocación e inmovilización del paciente no varían y la radiación generada en el Acelerador Lineal es correcta, la reconstrucción de la dosis no sería necesaria; sin embargo, en condiciones clínicas, la reconstrucción de la dosis es de gran utilidad para determinar la dosis realmente administrada y compararla con la dosis planificada. De esta manera la tomoterapia, permite cuantificar e incluso corregir las discrepancias de dosis, asegurando un excelente control de calidad del tratamiento ${ }^{16}$.
Conociendo la distribución de la dosis en el paciente representada en el TAC y que se corresponde con la dosis realmente recibida, la dosis reconstruida puede ser directamente comparada con la distribución de la dosis planificada representada en el TAC de planificación, proporcionando la definitiva verificación del tratamiento ${ }^{17}$. La reconstrucción de la dosis realizada con la tomoterapia podría reemplazar al tiempo requerido en las verificaciones dosimétricas utilizando maniquíes, que son consideradas en la metodología estándar para tratamientos con IMRT. Esta información es extraordinariamente importante y de gran utilidad debido a que permite corregir y/o adaptar el tratamiento con radiación incluso fracción a fracción, aportando una excelencia en seguridad y precisión ${ }^{18}$.

Un aspecto importante en la tomoterapia con radiación adaptada es la consideración del movimiento o del cambio de posición de los órganos o del target entre fracciones motivado por condiciones fisiológicas (respiración, repleción estructuras anatómicas). Lu y cols ${ }^{19}$ desarrollan un modelo de registro deformable, consistente en que los datos de tomoimagen diarios son deformados elásticamente al objeto de reproducir topográficamente las imágenes del TAC de planificación y una vez que dichos datos han sido computarizados se obtiene una distribución de dosis asociada a cada fracción y realizando dicho 


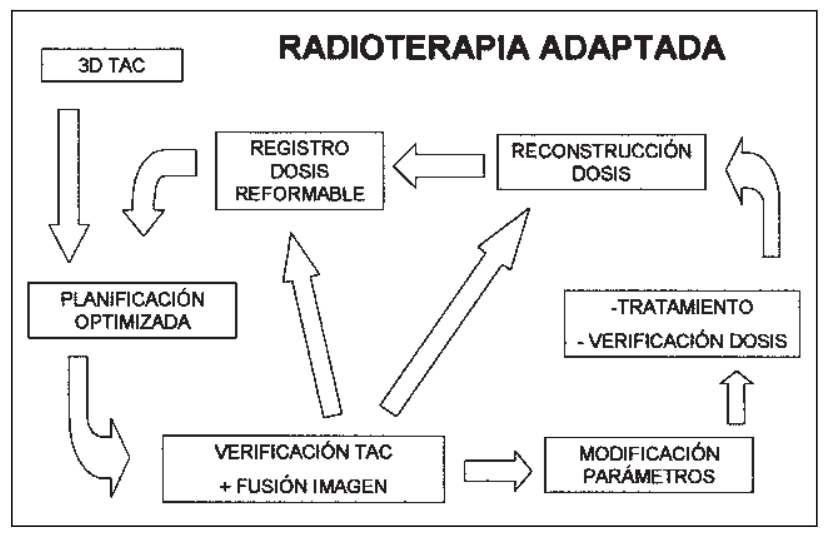

Figura 3. Algoritmo metodológico que define el proceso de radioterapia adaptada.

procedimiento en todas las fracciones de tratamiento, se obtiene la distribución de dosis "deformable" que se corresponde con la verdadera dosis administrada en cada órgano ${ }^{20}$.

Todos estos procedimientos, interrelacionados y retroalimentados, definen lo que se ha llamado la tomoterapia con radiación adaptada ${ }^{21}$ (Figura 3).

\section{Tomoterapia: interés clínico}

La causa de la recidiva tumoral local puede estar provocada no solamente por la administración insuficiente de la dosis de radiación prescrita en el tumor sino también por un cubrimiento geométrico inadecuado del tumor por la radiación. El movimiento del paciente o del órgano puede justificar una dosis insuficiente en la totalidad del target, y suponer un obstáculo para la correcta utilización de la radiación con intensidad modulada que utiliza haces de radiación no uniformes ${ }^{22}$. La definición de márgenes estrechos de seguridad alrededor del target (GTV, CTV), y el empleo de haces estrechos de radiación, (beamlets), pueden ser la causa de distribuciones de dosis no uniformes o inadecuadas en la periferia e incluso dentro del target si no existe la precisión adecuada, resultando un fallo local o una toxicidad no deseados.

La tomoterapia helicoidal proporciona una flexibilidad a propósito de los ángulos de incidencia de los haces de radiación, habida cuenta que es posible administrar radiación en ángulos completos de $360^{\circ}$ debido a que el gantry rota completamente alrededor del paciente al mismo tiempo que la mesa se mueve longitudinalmente, de manera que es posible obtener conformaciones geométricas que eviten la radiación de los órganos críticos ${ }^{23}$ a la vez que no compromete la administración de una dosis en el target.

La tomoterapia utiliza haces de radiación coplanares en todas las direcciones, existiendo la posibilidad de emplear un "bloqueo completo" que previene la incidencia del haz primario a través de una región a riesgo de manera que solo recibiría dicha área una irradiación dispersa o un "bloqueo de entrada" que permite que el haz salga a través de la región a riesgo después de atravesar el target. Esta capacidad de bloqueo reduce las limitaciones referentes a la disponibilidad únicamente de haces coplanares y de la no disponibilidad de electrones, además de proteger estructuras radiosensibles. La utilización de haces múltiples coplanares, produce una dispersión de la radiación en el tejido depositándose una dosis integral en un área de tejido normal más extensa; el modelo de Lyman que estudia la relación volumen-dosis y la probabilidad de complicación en tejido normal (NTCP) sugiere que la administración dispersa de una dosis más baja a un volumen mayor podría reducir la incidencia de complicaciones en tejido normal para una dosis integral dada administrada en el target $\mathrm{o}$ incrementar la probabilidad de control tumoral a la misma. La tomoterapia podría ser capaz de restringir la administración de radiación a través de ciertas trayectorias de manera que podría evitar estructuras sensibles adyacentes al target.

El tratamiento de tomoterapia mediante IMRT adaptada y guiada por imagen ha sido aceptada por la Food and Drug Administration (FDA), y, desde el punto de vista clínico es especialmente atractiva teniendo en cuenta su versatilidad, no existiendo limitaciones condicionadas por el tamaño de la lesión, número de lesiones, localización anatómica, además de ser una posible alternativa como tratamiento de rescate en recidivas tumorales en áreas previamente irradiadas.

\section{Tomoterapia. Revisión de la literatura. Experiencia clínica}

\section{Patología ORL}

Harari y cols ${ }^{24}$ incluyen un total de 20 pacientes con tumores de orofaringe y comparan la confor- 
mación de dosis en el target y en los tejidos normales adyacentes, utilizando tomoterapia helicoidal (51 haces proyectados por rotación con un mínimo de 20 rotaciones) versus IMRT convencional (7 campos) teniendo previsto administrar una dosis a PTV de 66-70 Gy, glándula parótida 20 Gy y médula espinal $35 \mathrm{~Gy}$, observando una mejoría en la distribución de dosis a PTV con una reducción de hasta el 50\% de la dosis en parótida contralateral, médula espinal y cavidad oral con tomoterapia helicoidal.

\section{Patología pulmonar}

La Universidad de Wisconsin justifica la utilización de tomoterapia helicoidal en el desarrollo de estudios de escalada de dosis por fracción en carcinoma no microcítico de pulmón ${ }^{25}$, y publica posteriormente los resultados de un estudio que incluye 5 pacientes con carcinoma no microcítico de pulmón, estadío III, inoperable, comparando la dosis de radiación en tejidos normales (pulmón, esófago, médula espinal) con tomoterapia helicoidal versus radioterapia convencional. La tomoterapia helicoidal reduce la dosis en tejido pulmonar normal, cuando se analiza la dosis normalizada media (NTD) y el volumen pulmonar que recibe una dosis superior a 20 Gy (V20) en aproximadamente $30 \%$ y $22 \%$ respectivamente $^{26}$. Si se considera la dosis recibida por médula espinal y esófago, observa que las dichas estructuras no son dosis limitantes con la tomoterapia helicoidal.

\section{Tumores prostáticos}

La deformación en la morfología de la próstata y del recto debido a la acumulación de gas y heces en el recto y de orina en la vejiga puede cambiar diariamente, en cada fracción de tratamiento, e influir en la dosis absorbida en el target y en las estructuras normales (vejiga, recto, intestino).

Byme y $\operatorname{cols}^{27}$ evalúa el impacto de la deformación del recto y de la próstata en la distribución de dosis administrada mediante controles diarios con tomoimagen (megavoltaje) y refiere que es necesario modificar las coordenadas lateral, supero-inferior y antero-posterior en $2.3,1.7$ y $2.6 \mathrm{~mm}$ respectivamente por discrepancias entre fracciones y observan que en más del 95\% de las ocasiones la línea de iso- dosis del 100\% incluye la próstata y vesículas seminales, confirmando que un margen de $10 \mathrm{~mm}$ alrededor de la próstata en todas las direcciones excepto 5 $\mathrm{mm}$ en el margen posterior es suficiente cuando se utiliza tomoterapia helicoidal; sin embargo, la deformación en el recto indica que el recto deformado recibiría una alta dosis de radiación en el $90 \%$ de las fracciones administradas. La tomoterapia, mediante su procedimiento metodológico de radioterapia adaptada, permitiría evitar anticipadamente dicho incremento de dosis en el recto, manteniendo la dosis prescrita en próstata y vesículas seminales, mediante la oportuna verificación y corrección si procede.

Langen y cols ${ }^{28}$ observan que la deformación diaria del recto provoca histogramas dosis-volumen diarios significativamente diferentes de los planificados y confirman que la dosis que recibe el volumen rectal es tanto más alta cuanto mayor es el contenido de gas del recto.

\section{Tumores del sistema nervioso}

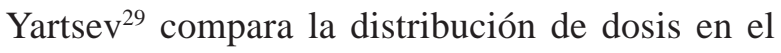
target (tumor) en los órganos a riesgo (globos oculares, tronco cerebral, tejido cerebral normal) en 12 pacientes con diferentes lesiones cerebrales (media: 4.6 c.c.) incluyendo meningiomas, neurinomas del acústico y adenomas de hipófisis, administrando una dosis media de 56 Gy en 28 fracciones, con tomoterapia helicoidal, radioterapia estereotáxica, radioterapia conformada 3D y radioterapia de intensidad modulada (IMRT) y concluye que la tomoterapia helicoidal mejora la distribución uniforme de la dosis en el target con similar distribución de dosis en los órganos a riesgo comparada con el resto de técnicas utilizadas.

Otra indicación especialmente atractiva para la tomoterapia es la irradiación craneo-espinal (30), que presenta una serie de ventajas respecto a la irradiación convencional:

1. Permite mayor comodidad para el paciente pudiendo ser tratado en posición de decúbito supino.

2. Es posible la irradiación de todo el volumen sin incertidumbres en las zonas de unión de campos.

3. La tomoimagen permite corregir y detectar posibles desalineaciones diarias del paciente. 


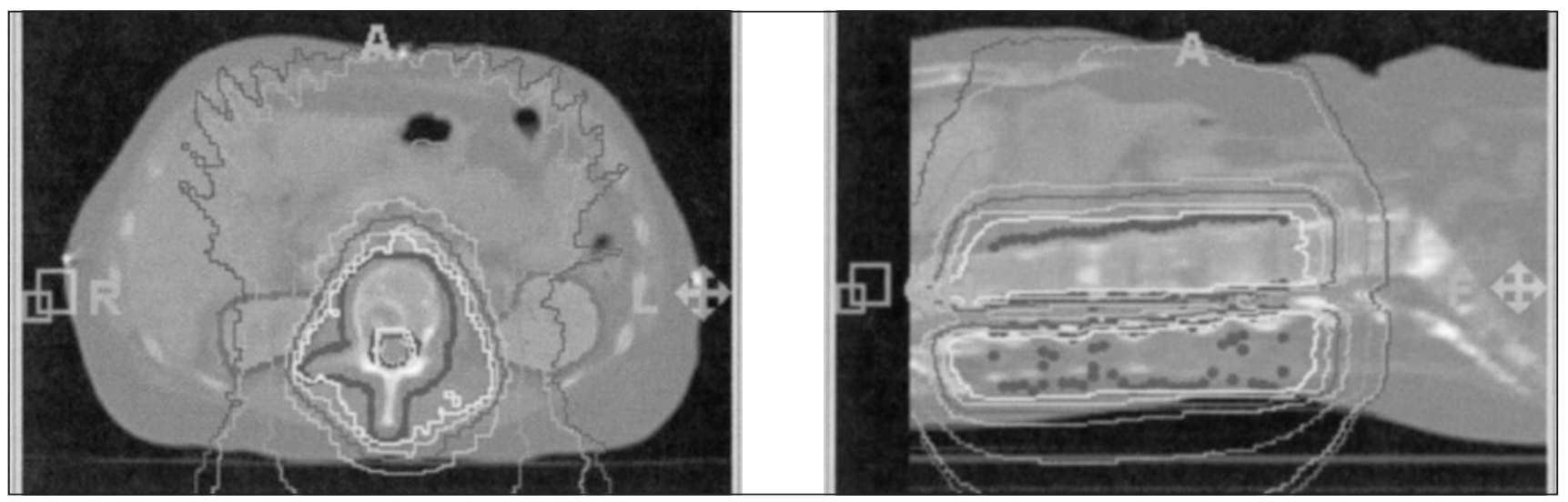

Figura 4. Reirradiación espinal (Tomotheray, Madison).

4. Es posible realizar el tratamiento de sobreimpresión del tumor primario, de forma sincrónica, mediante tratamientos altamente conformados, acortando la duración del mismo.

\section{Tomoterapia: aplicaciones de interés clínico}

La tomoterapia, permite el tratamiento de lesiones intra y extracraneales, que por su tamaño y localización, exigen una gran seguridad y precisión en su localización (Figura 4).

Asimismo, se trata de una atractiva técnica para el tratamiento de lesiones tumorales localizadas en órganos críticos (hígado, pulmón) en los que la radiación muchas veces está condicionada por la tolerancia limitada en función de la dosis acumulada por la totalidad del órgano.

\section{Radioterapia intracraneal}

Puede ser una alternativa al tratamiento con radiocirugía estereotáxica (dosis única) utilizada en lesiones intracraneales pequeñas $(5 \mathrm{~mm}-40 \mathrm{~mm})$, circunscritas, bien delimitadas y a la radioterapia estereotáxica fraccionada, utilizada en lesiones de mayor tamaño (> $45 \mathrm{~mm}$ ), mal delimitadas, de morfología irregular y próximas a las estructuras radiosensibles.

\section{Radioterapia hepática}

La radioterapia ha desempeñado un mínimo papel en el tratamiento potencialmente curativo de los pa- cientes con patología tumoral hepática (metástasis, hepatocarcinoma), fundamentalmente debido a la limitada radiotolerancia del hígado, estimada en un $5 \%\left(\mathrm{TD}_{5}\right)$ con $25 \mathrm{~Gy}$ y $50 \%\left(\mathrm{TD}_{50}\right)$ con 35 Gy si se irradia la totalidad del hígado (1.8-2.0 Gy/fracción) ${ }^{31}$.

Recientes estudios clínicos desarrollados en metástasis hepáticas (1-3 metástasis), utilizando técnicas estereotáxicas y empleando fraccionamientos alterados del tipo dosis única (14-26 Gy), o hipofraccionamientos (3 dosis de 10-20 Gy/fracción) han comunicado excelentes tasas de control local que oscilan entre el 81 y el 94\% (32, 33). Asimismo, tumores hepáticos primarios (hepatocarcinoma) tratados con una dosis total de 30 Gy en 5 fracciones o una dosis total de 24 Gy en 1-3 fracciones han presentado tasas de control local por encima del $94 \%$ con toxicidad aceptable.

\section{Radioterapia pulmonar}

En los últimos años, dos series han publicado resultados preliminares en carcinoma no microcítico de pulmón (CNMP), estadío precoz (T1-T2 N0) utilizando una dosis única de 20-30 Gy (34) comunicando una tasa de control local del $80 \%$ y $100 \%$ respectivamente. Timmerman y cols ${ }^{35}$ publican una tasa de control local tumoral del $83 \%$ en 37 pacientes con CNMP precoz, tratados con 3 fracciones de 820 Gy (estudio Fase I de escalada de dosis: 24-60 Gy dosis total) dato reproducido en diferentes series, utilizando entre 3 a 8 fracciones de 10-15 Gy/fracción ${ }^{33}$. 


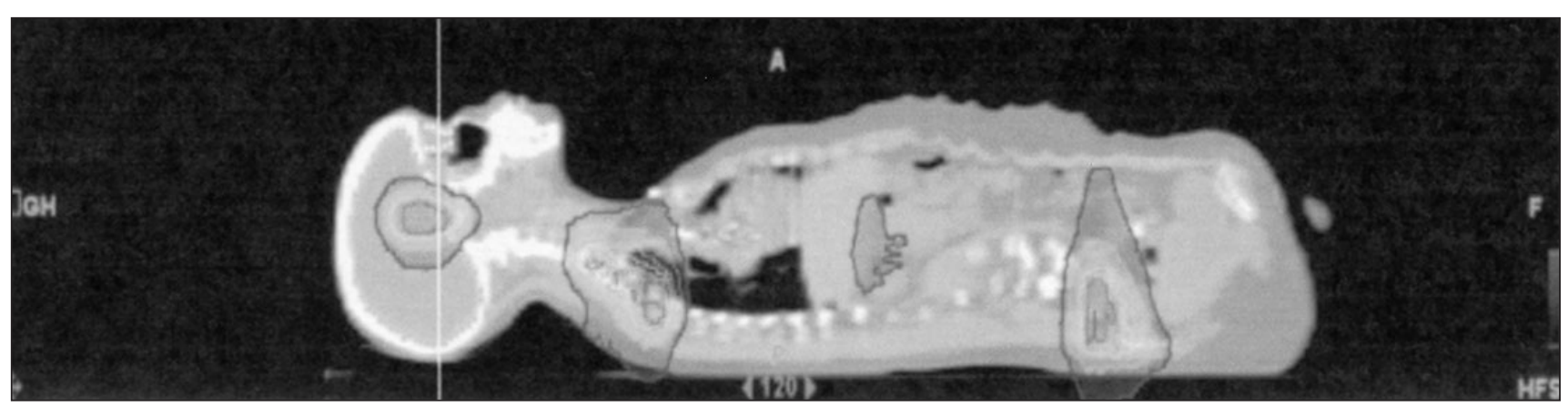

Figura 5. Distribución de dosis en irradiación craneoespinal. (London Regional Cancer Center, Canada)

Otros grupos han comunicado la factibilidad y tolerancia del tratamiento de metástasis pulmonares (1-3 metástasis) empleando similares programas de dosis única o dosis hipofraccionada con tasas de control del $85-100 \%$ siendo la toxicidad grado III (neumonitis) observada con técnicas estereotáxicas y dosis única (15-30 Gy) ha oscilado entre 0-4\% y con dosis hipofraccionada (3-8 fracciones) ha sido del $0-7 \%{ }^{35}$.

En general, diferentes aspectos han motivado la reciente incorporación a la práctica clínica de la tomoterapia como una nueva modalidad de tratamiento con radiaciones ionizantes, y que pueden ser de elección respecto a otras alternativas:

\section{- Multicentricidad}

- Misma área anatómica:

* Metástasis hepáticas.

* Metástasis pulmonares.

* Metástasis cerebrales.

- Diferente área anatómica:

* Regiones ganglionares afectas (supra y/o infradiafragmática).

* Metástasis multigeográficas (multiviscerales) (Figura 5).

\section{- Versatilidad y simplicidad:}

- Volúmenes amplios:

* Irradiación corporal total

* Irradiación total médula osea.

* Irradiación craneo-espinal.

- Volúmenes moderados:

* Tumores otorrinolaringológicos.

* Linfomas (irradiación ganglionar supra/infradiafragmática).

\section{- Volúmenes reducidos:}

* Patología intra/extracraneal con criterio para radiocirugía estereotáxica $<3.5 \mathrm{~cm}$ diámetro.

- Multimodalidad: Tratamientos combinados:

- Quimio-radioterapia:

* Adyuvante, neoadyuvante, radical.

* Nuevos fármacos.

* Ensayos clínicos: nuevos protocolos.

\section{- Movilidad anatómica:}

- movimientos respiratorios: pulmón, abdomen.

- condiciones fisiológicas viscerales: próstata (replección de vejiga, recto).

\section{- Nuevos protocolos radioterapia:}

- Escalada de dosis de radiación.

- Fraccionamientos alterados. (fraccionamiento acelerado, alta dosis única, hiperfraccionamiento, hipofraccionamiento, "boost concomitante").

- Re-irradiación de rescate.

\section{- Conformación estructuras radiosensibles}

\section{Conclusiones}

La tomoterapia helicoidal es considerada como un tratamiento con radiación de intensidad modulada guiada por imagen ${ }^{36}$. Un TAC de megavoltaje instalado en la unidad de radiación hace posible la verificación mediante TAC inmediatamente antes del tratamiento. Las imágenes de TAC pueden ser fusionadas automáticamente con el TAC de planificación y determinar la morfología en 3D y la posición del 
target antes de la radioterapia, permitiendo utilizar dicha información para ajustar posibles parámetros del paciente. Una tomoimagen en el momento del tratamiento puede ser utilizada como base para la reconstrucción de la dosis recibida por el paciente. La reconstrucción de la dosis permite que la dosis administrada puede ser superpuesta en el TAC correspondiente a la sesión de tratamiento y comparada con la distribución de la dosis planificada representada en el TAC de planificación. Si la anatomía ha sido alterada un registro deformable de la dosis puede ser utilizado para representar mediante mapeo la distribución de la dosis administrada respecto a la distribución de dosis planificada. El registro de la dosis con o sin registro deformable permitirá que la dosis administrada sea modificada en tratamientos sucesivos. Este conjunto de procesos de verificación que tiene como objetivo administrar correctamente la dosis a lo largo del tratamiento se ha definido como radioterapia adaptada.

\author{
Correspondecia: \\ Dr. J. M. Delgado \\ Dpto. Radiofísica \\ Instituto Madrileño de Oncología \\ C/ Emilio Vargas, 16 \\ E-28043 Madrid \\ E-mail: jmdelgado@grupoimo.com
}

\section{Bibliografía}

1. Jaffray DA, Drake DG, Moreau M, et al. A radiographic and tomographic imaging system integrated into a medical linear accelerator for localization of bone and softissue targets. Int J Radiat Oncol Biol Phys 1999; 45: 773-789.

2. Martínez AA, Yan D, Lockman D, et al. Improvement in dose escalation using the process of adaptive radiotherapy combined with three-dimensional conformal or intensitymodulated beams for prostate cancer. Int J Radiat Oncol Biol Phys 2001; 50: 1226-1234.

3. Siewerdsen JH, Jaffray DA. Cone-beam computed tomography with a flat-panel imager: Magnitude and effects of x-ray scatter. Med Phys 2001: 28: 220-231.

4. Mackie TR, Holmes T, Swerdloff S, et al. Tomotherapy: a new concept for the delivery of dynamic conformal radiotherapy. Med Phys 1993: 20: 1709-19

5. Olivera G, Shepard DM, Ruchala K, et al. Tomotherapy. In: Van Dyk J, editor. Modern technology of radiation oncology. Madison, WI: Medical Physics Publishing; 1999.
6. Mackie TR, Balog J, Ruchala K, et al. Tomotherapy. Semin Radiat Oncol 1999; 9: 108-117.

7. Mackie TR, Kapatoes J, Ruchala K, et al. Image guidance for precise conformal radiotherapy. Int. J. Radiation Oncology Biol. Phys. 2003: 56, N. 1: 89-105, 2003.

8. Ruchala K. Megavoltage computed tomography for tomotherapy verification. In: Dep. Of Medical Physics. Madison: 1999. University of Wisconsin.

9. Jeraj R, Mackie TR, Balog J, et al. Radiation characteristics of helical tomotherapy. Med. Phys. 31 (2), February 2004. 396.

10. Ruchala KJ, Olivera G, Kapatoes JM, et al. Megavoltage CT image reconstruction during tomotherapy treatments. Phys. Med. Biol. 2000; 45: 3545-3562. Printed in the UK.

11. Ruchala KJ, Olivera G, Schloesser EA, et al. Megavoltage CT on a tomotherapy system. Phys. Med. Biol., 1999 44: 2597-2621. Printed in the UK.

12. Ruchala KJ, Olivera G, Kapatoes JM. Limited-data image registration for radiotherapy positioning and verification. Int. J. Radiation Oncology Biol. Phys, 2002; 54: 592-605.

13. Kapatoes JM, Olivera G, Reckwerdt PJ, et al. Delivery verification in sequential and helical tomotherapy. Phys. Med. Biol., 1999; 44:1815-1841. Printed in the UK.

14. Kapatoes JM, Olivera G, Ruchala K, et al. On the verification of the incident energy fluence in tomotherapy IMRT. Phys Med Biol 2001; 46: 2953-2965.

15. Ruchala KJ, Olivera G, Kapatoes JM, et al. Megavoltage CT image reconstruction during tomotherapy treatments. Phys Med Biol 2000; 45: 3545-3562.

16. Kapatoes JM, Olivera G, Ruchala K, et al. A feasible method for clinical delivery verification and dose reconstruction in tomotherapy. Med Phys 2001; 28: 528-542.

17. Kapatoes JM, Olivera G, Balog JP, et al. On the accuracy and effectiveness of dose reconstruction for tomotherapy. Phys. Med. Biol., 2001; 46: 943-966.

18. Wu C, Jeraj R, Olivera G, et al. Re-optimization in adaptive radiotherapy. Phys. Med. Bio., 2002; 47: 3181-3195.

19. Lu W, Chen M, Olivera G, et al. Fast deformable registration using calculus of variations- 45 AAPM Meeting, San Diego CA, August 2004.

20. Yan D, Wong J. Adaptive modification of treatment planning to minimize the deleterious effects of treatment setup errors. Int J Radiat Oncol Biol Phys 1997; 38: 197-206.

21. Ruchala K, Olivera G. Adaptive IMRT with Tomotherapy. RT image. 2001; 14: No 25, 12-16.

22. Yu CX, Jaffray DA, Wong JW. The effects of intra-fraction organ motion on the delivery of dynamic intensity modulation. Phys Med Biol 1998; 43: 91-104.

23. Aldridge JS. Tomographic patien registration and conformal avoidance tomotherapy. In Dept. of Medical Physics; 1999. Madison: University of Wisconsin.

24. Harari PM, Jaradat HA, Connor NP, et al. Refining Target Coverage and Normal Tissue Avoidance with Helical Tomotherapy vs Linac-Based IMRT for Oropharyngeal Cancer. Procc ASTRO, 2004, abstr 51. 
25. Mehta M, Scrimger R, Mackie R, et al. A new approach to dose escalation in non-small-cell lung cancer. Int. J. Radiation Oncology Biol. Phys. 2001; 49: No 1, 23-33.

26. Scrimger R, Tomé WA, Olivera G, et al. Reduction in Radiation Dose to Lung and Other Normal Tissues Using Helical Tomotherapy to Treat Lung Cancer, in Comparison to Conventional Field Arrangements. Am J. Clin Oncol (CCT). 2003; 26(1): 70-78.

27. Byrne T, Chester R, Stephen M, et al. Daily Image-Guided Adaptive Radiotherapy for Prostate Cancer Using Helical Tomotherapy. Procc ASTRO, 2004, abstr 2457.

28. Langen K, Poole D, Zhang Y, et al. Using Daily MVCT Images To Calculate Daily Rectal Dose Volumen Histograms In Prostate Cancer Patients Treated With A Helical TomoTherapy Unit. Procc ASTRO, 2004, abstr 2456.

29. Yartsev S, Kron T, Cozzi L, et al. Tomotherapy planning of small brain tumours. Radiotherapy and Oncology. 2005; 74: 49-52.

30. Bauman G, Yartsev S, Coad T, et al. Helical tomotherapy for craniospinal radiation. The British Journal of Radiology, 2005; 78: 548-552.
31. Greco C, Catalamo G, Di Grazie A, Orecchia R. Radiotherapy of liver malignancies from whole liver irradiation to stereotactic hypofractionated radiotherapy. Tumori. 2004; 90: 73-79.

32. Blomgren H, Lax I, Göranson H, et al. Radiosurgery for Tumors in the Body: Clinical Experience Using a New Method. Journal of Radiosurgery, 1998; Vol. 1. No. 1: 63-74.

33. Song D, Kavanagh B, Benedict S, et al. Stereotactic Body Radiation Therapy. Rationale, Techniques, Applications, and Optimization. Oncology, 2004; 18: 1419-1430.

34. Hara R, Itami J, Kondo T, et al. Stereotactic single high dose irradiation of lung tumors Ander respiratory gating. Radiother Oncol. 2003; 63: 159-163.

35. Timmerman R, Papiez L, McGarry R, et al. Extracranial Stereptactoc Radioablation. Results of a Phase I Study in Medically Inoperable Stage I non-small Cell Lung Cancer. Chest 2003; 124: 1946-1955.

36. Beavis AW. Is tomotherapy the future of IMRT? The British Journal of Radiology, 2004; 77: 285-295. 